Proc. of the 11 Int. School on Theoretical Physics Symmetry and Structural Properties of Condensed Matter, Rzeszów 2014

\title{
Matrix Representation of Constants of Motion for One-Dimensional Heisenberg Magnet
}

\author{
P. JAKUBCZYK*, M. LABUZ AND T. LULEK \\ Department of Theoretical Physics, Faculty of Mathematics and Natural Sciences, University of Rzeszow, \\ Pigonia 1, 35-310 Rzeszów, Poland
}

\begin{abstract}
We demonstrate an exact diagonalization of the one-dimensional Heisenberg magnet in terms of algebraic Bethe Ansatz. We point out, by a polynomial expansion of the transfer matrix with respect to spectral parameter, a complete set of observables for classification of all eigenstates. We introduce an application of our approach on the example of the Heisenberg magnet consisting of four qubits, including its constants of motion, density matrices and complete classification of eigenstates.
\end{abstract}

DOI: 10.12693/APhysPolA.128.176

PACS: 03.65.Aa, 75.10.Jm

\section{Introduction}

The isotropic XXX Heisenberg spin chain is one of the simplest quantum integrable systems. Its integrability means that one can find sufficiently many constants of motion, which completely classify all eigenstates of the system. Baxter [1] showed that the monodromy matrices for different spectral parameters commute, i.e. $[M(\lambda), M(\mu)]=0$, while Sutherland [2] proved that the transfer matrix commutes with the Hamiltonian $[H, T(\lambda)]=0$. Thus, we are able to find constants of motion in terms of the transfer matrix $T$. In order to go through this problem, one introduces auxiliary space $V=\mathbb{C}^{2}$, and greater working space $\mathcal{H} \otimes V$, as the arena of algebraic Bethe Ansatz (ABA) [3]. Next, if we get the trace of the monodromy matrix in the working space, we obtain a polynomial of degree $N$ in terms of $\lambda$ of the form [3]:

$$
T(\lambda)=2 \lambda^{N} I_{\mathcal{H}}+\sum_{n=0}^{N-2} \lambda^{n} \widehat{T}_{n},
$$

where $I_{\mathcal{H}}$ stands for the identity matrix in the Hilbert space $\mathcal{H}$ of the model, and $\widehat{T}_{n}$ are constants of motion.

The main aim of the paper is to present explicit matrix construction of these constants of motion for the onedimensional Heisenberg magnet consisting of four qubits, based directly on Eq. (1). We point out that the coefficients $T_{n}$ of the transfer matrix $T$, seen as a polynomial with respect to $\lambda$, provide a matrix representation of a complete set of commuting observables which uniquely classify eigenstates of the Heisenberg model, exactly along the famous recipe of Dirac [4]. This approach can be considered as an alternative of original Bethe Ansatz [5].

* corresponding author; e-mail: pjakub@ur.edu.pl

\section{The model}

Let us consider the Heisenberg model consisting of $N$ qubits. The Hilbert space of such a system has the form

$$
\mathcal{H}=\left(\mathbb{C}^{2}\right)^{\otimes N},
$$

where $\mathbb{C}^{2}$ is a local Hilbert space of the qubit. In order to introduce a computational basis of the system let us consider the set $\tilde{N}=\{j=1,2, \ldots, N\}$ of nodes of the magnetic ring, and the set $\tilde{2}=\{0,1\}$ of single-node states of a qubit. Then the basis elements can be written as

$$
|f\rangle \equiv\left|i_{1}, i_{2}, \ldots, i_{N}\right\rangle, \quad i_{j} \in \tilde{2}, \quad j \in \tilde{N} .
$$

All linear combinations of basis elements (3) with complex coefficients belong to the Hilbert space (2). The Heisenberg Hamiltonian is an algebra element of the symmetric group, therefore its action on magnetic configurations (3) does not change the number of spin deviations. Thus, the space $\mathcal{H}$ of all quantum states of the Heisenberg magnet, with $\operatorname{dim} \mathcal{H}=2^{N}$, decomposes as

$$
\mathcal{H}=\sum_{r=0}^{N} \oplus \mathcal{H}^{r},
$$

into subspaces $\mathcal{H}^{r}, \operatorname{dim} \mathcal{H}^{r}=\left(\begin{array}{l}N \\ r\end{array}\right)$, with the fixed number $r$ of Bethe pseudoparticles (spin deviations). The Hamiltonian which contains only exchange interactions between spins at neighbour sites (but no annihilation or creation of a spin on chain sites) has the form

$$
\widehat{H}=\frac{1}{2} \sum_{n=1}^{N}\left(P_{n n+1}-I_{n n+1}\right),
$$

where, according to boundary conditions, $n=n+m N$, $m \in \mathbb{Z}$. The permutation operator $P_{n n+1}$ exchanges two spin states at sites $n$ and $n+1$. In our case of the spin $s=\frac{1}{2}$ this operator, according to the Dirac identity

$$
P_{n n+1}=\frac{1}{2}\left(\boldsymbol{\sigma}_{n} \boldsymbol{\sigma}_{n+1}+I_{n n+1}\right),
$$

can be represented in terms of the Pauli matrices $\boldsymbol{\sigma}=$ $\left(\sigma^{1}, \sigma^{2}, \sigma^{3}\right)$. Here, the identity matrix $I_{n n+1}$ acts in the space $\left(\mathbb{C}^{2}\right)_{n} \otimes\left(\mathbb{C}^{2}\right)_{n+1}$. 


\section{The transfer matrix}

Technique of ABA bases on the space with dimension $\operatorname{dim} 2^{N+1}$, and it requires the introduction of an auxiliary qubit to reach the form

$$
\mathcal{H} \otimes \mathbb{C}^{2} \cong\left(\mathbb{C}^{2}\right)^{\otimes(N+1)} .
$$

In such an extended space, there acts the Lax operator

$$
L_{n}(\lambda)=\left(\lambda-\frac{\mathrm{i}}{2}\right) I \otimes I_{a}+\mathrm{i} P_{n, a},
$$

where $P_{n, a}$ is the permutation operator between the spaces $\left(\mathbb{C}^{2}\right)_{j}$ and $\left(\mathbb{C}^{2}\right)_{a}$. The form of the Lax operator in (8) is given by applying the Dirac identity between the product of two single-node spin operators $\left(\mathbf{s}_{\mathbf{n}}, \mathbf{s}_{\mathbf{a}}\right)$ and two-node permutation operator $P_{n, a}$ (cf. Eq. (6)).

Next, one introduces the monodromy matrix

$$
\widehat{M}(\lambda):=\widehat{L}_{N}(\lambda) \ldots \widehat{L}_{1}(\lambda)=\left(\begin{array}{cc}
\widehat{a}(\lambda) & \widehat{B}(\lambda) \\
\widehat{C}(\lambda) & \widehat{D}(\lambda)
\end{array}\right),
$$

where $\widehat{a}(\lambda)$ and $\widehat{D}(\lambda)$ are weight operators, whereas $\widehat{B}(\lambda)$ and $\widehat{C}(\lambda)$ are creation and annihilation operators of a single Bethe pseudoparticle, respectively. Taking trace over the auxiliary space of the monodromy matrix one obtains the transfer matrix

$$
\widehat{T}(\lambda)=\widehat{a}(\lambda)+\widehat{D}(\lambda),
$$

which has a polynomial representation with respect to $\lambda$ of the form given in (1). Operator coefficients of this polynomial constitute the maximal set of commuting observables of the model. These observables can be used for the complete classification of all eigenstates of the system.

\section{Exact diagonalization of magnet with four qubits}

In this section we propose the procedure of an exact diagonalization of the Heisenberg Hamiltonian for the magnet consisting of four qubits. As a subspace of the Hilbert space with $r$ spin deviations is invariant under the action of the Hamiltonian (5), we restrict ourselves to the subspace with $r=2$ spin deviations (cf. Eq. (4)). This allows us to diminish the size of the transfer matrix from $2^{4}=16$ to $\left(\begin{array}{c}N \\ r\end{array}\right)=\left(\begin{array}{l}4 \\ 2\end{array}\right)=6$. It is worth to mention that calculations in the subspaces with different number of spin deviations $r$ can be performed analogously. Basing on the procedure described in Sect. 3 we find the transfer matrix of the form

$$
T(\lambda)=\left[\begin{array}{cccccc}
a & b & 0 & b^{*} & c & c \\
b^{*} & a & b & 0 & c & c \\
0 & b^{*} & a & b & c & c \\
b & 0 & b^{*} & a & c & c \\
c & c & c & c & a & 1 \\
c & c & c & c & 1 & a
\end{array}\right]
$$

where $a=2 \lambda^{4}+\frac{1}{8}+\lambda^{2}, b=-\lambda^{2}+i \lambda+\frac{1}{4}, c=-\lambda^{2}-\frac{1}{4}$, and $b^{*}$ denotes the complex conjugation of $b$. Considering the polynomial coefficients with the power of $\lambda$ (cf. Eq. (1)) we obtain three linearly independent (the whole number of constants of motion is 5) constants of motion

$$
T_{0}=\left[\begin{array}{cccccc}
1 / 8 & 1 / 4 & 0 & 1 / 4 & -1 / 4 & -1 / 4 \\
1 / 4 & 1 / 8 & 1 / 4 & 0 & -1 / 4 & -1 / 4 \\
0 & 1 / 4 & 1 / 8 & 1 / 4 & -1 / 4 & -1 / 4 \\
1 / 4 & 0 & 1 / 4 & 1 / 8 & -1 / 4 & -1 / 4 \\
-1 / 4 & -1 / 4 & -1 / 4 & -1 / 4 & 1 / 8 & 1 \\
-1 / 4 & -1 / 4 & -1 / 4 & -1 / 4 & 1 & 1 / 8
\end{array}\right]
$$

with eigenvalues $\operatorname{spec}\left(T_{0}\right)=\left(\frac{-7}{8}, \frac{-3}{8}, \frac{1}{8}, \frac{1}{8}, \frac{1}{8}, \frac{13}{8}\right)$,

$$
T_{1}=\left[\begin{array}{cccccc}
0 & \mathrm{i} & 0 & -\mathrm{i} & 0 & 0 \\
-\mathrm{i} & 0 & \mathrm{i} & 0 & 0 & 0 \\
0 & -\mathrm{i} & 0 & \mathrm{i} & 0 & 0 \\
\mathrm{i} & 0 & -\mathrm{i} & 0 & 0 & 0 \\
0 & 0 & 0 & 0 & 0 & 0 \\
0 & 0 & 0 & 0 & 0 & 0
\end{array}\right]
$$

with eigenvalues $\operatorname{spec}\left(T_{1}\right)=(-2,0,0,0,0,2)$, and

$$
T_{2}=\left[\begin{array}{cccccc}
1 & -1 & 0 & -1 & -1 & -1 \\
-1 & 1 & -1 & 0 & -1 & -1 \\
0 & -1 & 1 & -1 & -1 & -1 \\
-1 & 0 & -1 & 1 & -1 & -1 \\
-1 & -1 & -1 & -1 & 1 & 0 \\
-1 & -1 & -1 & -1 & 0 & 1
\end{array}\right]
$$

with eigenvalues $\operatorname{spec}\left(T_{2}\right)=(-3,1,1,1,3,3)$. It can be easily checked that they mutually commute i.e. $\left[T_{0}, T_{1}\right]=$ $\left[T_{1}, T_{2}\right]=\left[T_{2}, T_{0}\right]=0$. Applying standard quantum mechanical techniques one can calculate projection operators for each constant of motion and its eigenvalues. A projection operator of the $j$-th commutative operator $T_{j}$ into its eigenvalue $a$ is given by the formula

$$
P_{a}^{(j)}=\prod_{\substack{a^{\prime} \in \operatorname{spec}_{n d}\left(\widehat{T}_{j}\right) \\ a^{\prime} \neq a}} \frac{\widehat{T}_{j}-a^{\prime} I_{\mathcal{H}^{(2)}}}{a-a^{\prime}},
$$

where $\operatorname{spec}_{n d}\left(\widehat{T}_{j}\right)$ denotes non-degenerate spectrum of the operator $\widehat{T}_{j}$, and $I_{\mathcal{H}^{(2)}}$ stands for the identity operator in the Hilbert space $\mathcal{H}^{(2)}$ with two spin deviations.

Next, we are able to build density matrices of Bethe eigenstates of the model by appropriate products

$$
\rho=P_{a_{1}}^{(1)} \cdot P_{a_{2}}^{(2)} \cdots \cdot P_{a_{l}}^{(l)},
$$

where $l$ denotes the number of linearly independent mutually commuting operators, so that $P_{a_{j}}^{(j)}$ denotes the projector of the commuting operator $T_{j}$ into its eigenvalue $a_{j}$. All density matrices for the considered model are listed in Table I.

Product basis of our model consists of regular and doubly rarefied orbit of the group $C_{4}$, therefore one can easily apply the discrete Fourier transform. Preserving the order resulting from the tensor product of the single node basis we obtain the matrix representation of the discrete Fourier transform (Eq. (12)) 


$$
\mathrm{DFT}=\frac{1}{2}\left[\begin{array}{cccccc}
-\mathrm{i} & -1 & \mathrm{i} & 1 & 0 & 0 \\
-1 & 1 & -1 & 1 & 0 & 0 \\
\mathrm{i} & -1 & -\mathrm{i} & 1 & 0 & 0 \\
1 & 1 & 1 & 1 & 0 & 0 \\
0 & 0 & 0 & 0 & -\sqrt{2} & \sqrt{2} \\
0 & 0 & 0 & 0 & \sqrt{2} & \sqrt{2}
\end{array}\right]
$$

Using expectation value of the Hamiltonian, translation operator and the total spin, we present a full classification of the problem discussed in Table II.

\section{TABLE I}

Density matrices for the Heisenberg magnet with four qubits calculated in terms of constants of motion. Lower indices in $\rho$ indicate eigenvalues of appropriate constant of motion from which density matrix was built.

$$
\begin{aligned}
& \rho_{\left[-\frac{7}{8}, 0,1\right]} \\
& \frac{1}{2}\left[\begin{array}{cccccc}
0 & 0 & 0 & 0 & 0 & 0 \\
0 & 0 & 0 & 0 & 0 & 0 \\
0 & 0 & 0 & 0 & 0 & 0 \\
0 & 0 & 0 & 0 & 0 & 0 \\
0 & 0 & 0 & 0 & 1 & -1 \\
0 & 0 & 0 & 0 & -1 & 1
\end{array}\right] \\
& \frac{1}{4}\left[\begin{array}{cccccc}
1 & -1 & 1 & -1 & 0 & 0 \\
-1 & 1 & -1 & 1 & 0 & 0 \\
1 & -1 & 1 & -1 & 0 & 0 \\
-1 & 1 & -1 & 1 & 0 & 0 \\
0 & 0 & 0 & 0 & 0 & 0 \\
0 & 0 & 0 & 0 & 0 & 0
\end{array}\right] \\
& \rho_{\left[\frac{13}{8}, 0,3\right]} \\
& \frac{1}{12}\left[\begin{array}{cccccc}
1 & 1 & 1 & 1 & -2 & -2 \\
1 & 1 & 1 & 1 & -2 & -2 \\
1 & 1 & 1 & 1 & -2 & -2 \\
1 & 1 & 1 & 1 & -2 & -2 \\
-2 & -2 & -2 & -2 & 4 & 4 \\
-2 & -2 & -2 & -2 & 4 & 4
\end{array}\right] \\
& \begin{array}{c}
\rho_{\left[\frac{1}{8}, 2,1\right]} \\
\frac{1}{4}\left[\begin{array}{cccccc}
1 & \mathrm{i} & -1 & -\mathrm{i} & 0 & 0 \\
-\mathrm{i} & 1 & \mathrm{i} & -1 & 0 & 0 \\
-1 & -\mathrm{i} & 1 & \mathrm{i} & 0 & 0 \\
\mathrm{i} & -1 & -\mathrm{i} & 1 & 0 & 0 \\
0 & 0 & 0 & 0 & 0 & 0 \\
0 & 0 & 0 & 0 & 0 & 0
\end{array}\right]
\end{array} \\
& \rho_{\left[\frac{1}{8},-2,1\right]} \\
& \frac{1}{4}\left[\begin{array}{cccccc}
1 & -\mathrm{i} & -1 & \mathrm{i} & 0 & 0 \\
\mathrm{i} & 1 & -\mathrm{i} & -1 & 0 & 0 \\
-1 & \mathrm{i} & 1 & -\mathrm{i} & 0 & 0 \\
-\mathrm{i} & -1 & \mathrm{i} & 1 & 0 & 0 \\
0 & 0 & 0 & 0 & 0 & 0 \\
0 & 0 & 0 & 0 & 0 & 0
\end{array}\right] \\
& \rho_{\left[\frac{1}{8}, 0,-3\right]} \\
& \frac{1}{6}\left[\begin{array}{llllll}
1 & 1 & 1 & 1 & 1 & 1 \\
1 & 1 & 1 & 1 & 1 & 1 \\
1 & 1 & 1 & 1 & 1 & 1 \\
1 & 1 & 1 & 1 & 1 & 1 \\
1 & 1 & 1 & 1 & 1 & 1 \\
1 & 1 & 1 & 1 & 1 & 1
\end{array}\right]
\end{aligned}
$$

TABLE II

Exact diagonalization of the Heisenberg magnet consisting of four qubits in the subspace with two spin deviations. The second column $T_{0}, T_{1}, T_{2}$ represents eigenstates in terms of quantum numbers (eigenvalues) of the maximal set of mutually commuting observables. The third column presents appropriate eigenvalues of energy $E$, quasimomentum $k$ and total spin $S$.

\begin{tabular}{c|c|c|c|c|c|c}
\hline \hline No. & $T_{0}$ & $T_{1}$ & $T_{2}$ & $E$ & $k$ & $S$ \\
\hline 1 & $-7 / 8$ & 0 & 1 & -4 & 2 & 1 \\
2 & $-3 / 8$ & 0 & 3 & -2 & 2 & 0 \\
3 & $13 / 8$ & 0 & 3 & -6 & 0 & 0 \\
4 & $1 / 8$ & 2 & 1 & -2 & 1 & 1 \\
5 & $1 / 8$ & -2 & 1 & -2 & -1 & 1 \\
6 & $1 / 8$ & 0 & -3 & 0 & 0 & 2
\end{tabular}

\section{Conclusions}

We have shown that the procedure of the complete diagonalization of the Heisenberg Hamiltonian can be easily performed using the transfer matrix, an element in ABA approach. Operator coefficients of the transfer matrix become some operators whose spectra completely classify Bethe eigenstates, and uniquely admit their presentation as density matrices.

\section{References}

[1] R.J. Baxter, Ann. Phys. 70, 323 (1972).

[2] B. Sutherland, J. Math. Phys. 11, 3183 (1970).

[3] L.D. Faddeev, L.A. Takhtajan, J. Sov. Math. 24, 241 (1984).

[4] P.A.M. Dirac, Phys. Today 11, 32 (1958).

[5] H. Bethe, Z. Phys. 71, 205 (1931) (in German; English translation in: D.C. Mattis, The Many-Body Problem, World Sci., Singapore 1993, p. 689). 